\title{
Multi-Objective Optimization of Non-Axisymmetric Contoured Endwall for Axial Turbines
}

\author{
Pingting Chen ${ }^{2}$, Xueying $\mathrm{Li}^{1}$, Jing Ren $^{2}$ and Hongde Jiang ${ }^{2}$ \\ ${ }^{1}$ Department of Energy and Power Engineering, Tsinghua University, Beijing 100084, PR China. \\ lixy@tsinghua.edu.cn \\ ${ }^{2}$ Department of Energy and Power Engineering, Tsinghua University
}

\begin{abstract}
Non-axisymmetric contoured endwall can reduce aerodynamic loss in axial turbine if the endwall shape is well designed. Meanwhile, contouring of the endwall can change the value of the total heat flux through the endwall, mainly by changing the distribution of heat transfer coefficient and the endwall area. In this study, several optimized non-axisymmetric contoured endwall shapes are found in an annular cascade passage by a multi-objective optimization process to achieve both improved aerodynamic performance in the passage and more beneficial heat transfer characteristics on the endwall. The optimized contoured endwall designs were found and they are all with sunken area in the middle of the passage and risen area in the aft part of the passage near the suction side. In addition, the case with better heat transfer performance features higher areaaveraged heat transfer coefficient but with smaller total endwall area. NOMENCLATURES

$\begin{array}{ll}C_{a x} & \text { axial chord length } \\ h & \text { heat transfer coefficient } \\ \mathrm{HT} & \text { total heat flux } \\ k & \text { the exponent of the penalty term } \\ \mathrm{LE} & \text { leading edge } \\ \mathrm{Ma} & \text { Mach number } \\ N u & \text { Nusselt number } \\ \mathrm{NGV} & \text { nozzle guide vane } \\ \mathrm{P} & \text { pressure } \\ \text { Span } & \text { vane span } \\ \mathrm{St} & \text { Strouhal number } \\ \mathrm{TE} & \text { trailing edge } \\ \mathrm{TIT} & \text { turbine inlet temperature } \\ \mathrm{TPLC} & \text { total pressure loss coefficient } \quad\left(P_{t, \text { inlet }}-\right. \\ \left.P_{t}\right) /\left(P_{t, \text { inlet }}-P_{\text {outlet }}\right) & \\ \mathrm{U} & \text { velocity } \\ x & \text { axial coordinate } \\ y & \text { pitchwise coordinate } \\ \theta_{\text {pitch }} & \text { annular angle of one pitch } \\ w & \text { weight factor } \\ \triangle \mathrm{R} & \text { local change of radial height from the }\end{array}$

baseline case

$$
t
$$

\section{INTRODUCTION}

total
\end{abstract}

As one of the most important electricity and power equipment, gas turbines are facing higher cycle efficiency requirements and

Presented at International Gas Turbine Congress 2019 Tokyo,

November 17-22, Tokyo, Japan

Review Completed on, September 17, 2020 harder durability problems. On one hand, the averaged turbine inlet temperature (TIT) is raised higher with each successive gas turbine generations to achieve progressively higher efficiency values, which makes the TIT near the endwall higher; thus, increasing the cooling demand of the Nozzle Guide Vane (NGV) endwall and causing more durability problems on the endwall. On the other hand improving the efficiency of turbine can be an effective way to improve the overall thermal cycle efficiency for gas turbine. With secondary loss near the endwall amounting to more than $35 \%$ of the total loss [1] for high pressure low aspect ratio turbines, the reducing of the secondary loss is becoming an effective way to elevate the efficiency of the axial turbines.

Changing the endwall shape to non-axisymmetric contoured shape is a way to reduce secondary loss. With the objective to improving the aerodynamic performance in the passage, Hartland and Harvey et al. [2,3] employed an inverse design system to minimize the secondary flow deviations and the secondary kinetic energy of the passage vortexes in a blade passage by lifting the endwall near the airfoil pressure side and sinking the endwall near the suction side to reduce the pitchwise pressure gradient. Their design was based on sinusoidal shapes generated in the circumferential direction at several axial locations. Most of the following investigations on contoured endwall design combined a pitchwise shape function (mostly sinusoidal shapes) and a streamwise decay function to reduce pitchwise pressure gradients. However, T.J. Praisner et al. [4] proposed that using simple functions to parameterize the shape of the endwall has much restrictions in geometry. Instead, they parameterized the endwalls by locating free points on the endwalls. They combined a gradientbased optimization algorithm with CFD flow solver to systematically vary a free-form parameterization of the endwall. Such optimization methods are employed on conventional lift (Pack B) and high-lift blade designs (Pack D-F). With experimental validation, the predicted and measured row-loss reduction was $4 \%$ and $10 \%$ for Pack B, while $12 \%$ and $25 \%$ for Pack D-F.

With the high cooling demand on the NGV endwall, it is important to evaluate the heat transfer characteristics on the contoured endwall. Some investigators studied heat transfer characteristics of given non-axisymmetric contoured endwalls. Lynch et al. [5] carried out Nusselt number measurement on the contoured hub of the contoured-endwall passage and the flat hub of the flat-endwall passage. The contoured endwall shape was designed in [4]. They found that Heat transfer levels were reduced by $20 \%$ in regions of high heat transfer and increased in regions of low heat transfer with endwall contouring, as compared to the flat endwall. The total heat transfer was reduced by $2 \%$ with a $3.1 \%$ reduction in the averaged heat transfer coefficient value and a $1.3 \%$ increasing of endwall area value. 
Endwall contouring can be used to change the local heat transfer coefficient distribution and the total endwall area. Winkler [6] used ice formation method and obtained heat transfer reduction on the designed endwall. Then, he did further heat transfer optimization using CFD method based on the endwall shape designed by the ice formation. However, all the contoured endwalls designed in his study achieve their heat transfer reductions at the expense of an increased total pressure loss comparing to the flat endwall case. Thus, endwall designs with better aerodynamic performance may got worse heat transfer characteristics, vice versa.

Considering the demand for both improved aerodynamic performances in the passage and more beneficial heat transfer characteristics on the endwall, optimization study with both the aerodynamic performance and the heat transfer characteristics objectives should be a beneficial extension of current work. In this study, a multi-objective design optimization approach of the nonaxisymmetric contoured endwall, which is capable of reducing aerodynamic loss in the passage while still decreasing the total heat flux through the endwall, was developed. The Pareto front of the optimization problem was found, and several optimized endwall designs were studied to understand the physics.

\section{GEOMETRY AND PARAMETERIZATION}

The optimization process of the non-axisymmetric contoured endwall shape was carried out on the hub of an annular cascade passage based on a F-class NGV. The shroud of the passage was kept as annular. The geometry parameters and flow parameters of the cascade are listed in Table 1. The radial height of each location on the hub is determined by a loft surface going through several cut lines located on the hub. For the current study, 5 cut lines were evenly distributed across one pitch and the shape of each cut line is determined using the Bézier curve [7] by control points (see Figure 1) including free points and frozen points located on each line (green dots for free points and red dots for frozen points). The radial heights of the free points are adjustable during the optimization approach while the radial heights of the frozen points are fixed as the height of the baseline case. These frozen points (red dots) are to ensure the endwall G1 continuous at the junctions between the endwall contouring zone and the endwall zones upstream and downstream of the contouring zone. As shown in Figure 1, there are totally 30 free points and 20 frozen points on the endwall. Thus, the free parameters in the current optimization approach to determine the endwall shape are the values of the 30 free points' radial heights.

Table 1 Parameters of the Cascade

\begin{tabular}{c|c}
\multicolumn{2}{c}{ Table 1 Parameters of the Cascade } \\
\hline Parameter & Value \\
\hline$C_{a x}[\mathrm{~m}]$ & 0.0785 \\
Span $/ C_{a x}[-]$ & 0.8790 \\
$\theta_{\text {pitch }}[\mathrm{deg}]$ & 9 \\
Inlet total temperature[K] & 310 \\
${\left.\text { Inlet flow angle }{ }^{\circ}\right]}$ & 0 \\
${\text { Exit flow angle }\left[^{\circ}\right]}^{\circ}$ & 73 \\
Inlet Ma[-] & 0.0844 \\
Exit Ma[-] $^{\circ}$ & 0.2841 \\
Inlet turbulence intensity & $5 \%$ \\
\hline
\end{tabular}

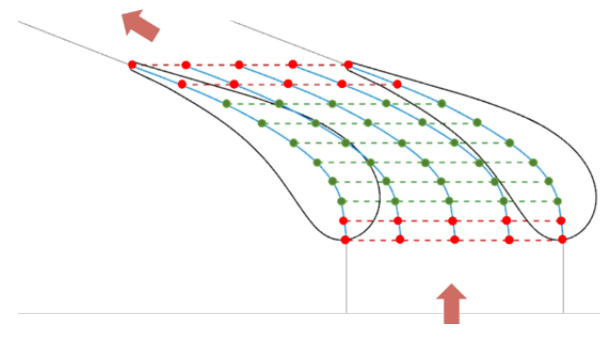

(a) Cut lines and control points

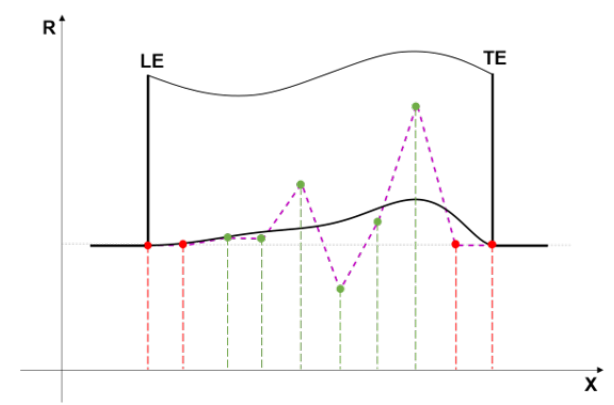

(b) Bézier curve by control points

Fig. 1 Shaping method from control points: (a) Cut lines(blue lines) and control points(green dots are free points and red dots are frozen points) on the endwall (b) A cut line determined using the Bézier curve [7] by control points

\section{COMPUTATIONAL SETUP AND CFD VALIDATION}

Figure 2 shows one of the computational domains with a contoured hub. The shape of the contoured hub is adjustable by different sets of the 30 free geometry parameters during the optimization process. Averaged total pressure was assigned at the mainstream inlets and averaged static pressure was specified at the mainstream outlets. The boundary conditions of the two side walls for each passage were set as periodic. The temperature of the hubs is fixed to be with a certain temperature difference from that of the mainstream inlet to calculate the heat flux distributions between the mainstream and the hub surface, thus, to derive the heat transfer coefficient distributions. All the other walls were non-slip, adiabatic walls.

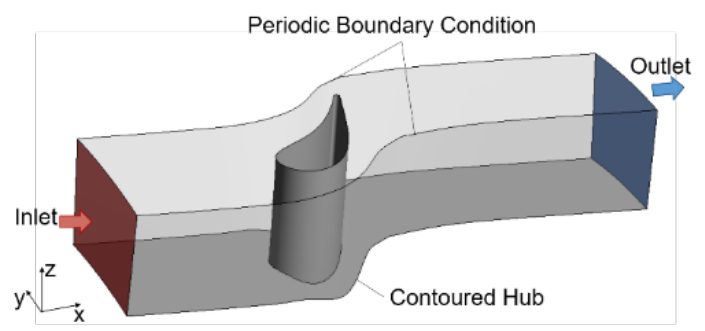

Fig. 2 Solution domain of the annular passage with contoured hub and un-contoured shroud

The CFD problems in the optimization process were solved by a commercial numerical solver with a three-dimensional, finitevolume method named FINE ${ }^{\mathrm{TM}} /$ Turbo. Depending on the CFD method validation, which will be described later, the SST model was employed.

Structured meshes were generated for the CFD simulations. Otype grids were used around the vane to get higher grid quality. The boundary layer regions on all the walls were refined to ensure values of $y+\sim 1$. With grid independency test, a meshing method for the 1.2 million grid points is employed during the optimization process.

The CFD method used in the current optimization approach is validated against the measured data by Li et al. [8]. This experiment was done in a linear cascade based on the same F-class NGV as 
used in the current optimization process. The static pressure distribution on the endwall is measured by pressure sensors connected to small holes located on the endwall surface, and the heat transfer coefficient distribution is measured using temperature sensitive paint (TSP) technique. Similar meshing methods and boundary conditions as those used in the current optimization process were employed to generate the grid points and to assign the boundary conditions for the validation case with the linear cascade.

Figure 3 compares the static pressure coefficient $\left(P / P_{t}\right)$ distributions on the endwall, both measured and CFD-predicted, using SST model, S-A model and $k-\varepsilon$ model. The parameter plotted here is the local static pressure, $P$, normalized by the approach flow total pressure, $P_{t}$. Little differences in the predicted pressure distributions on the endwall are found among these three different turbulence models and also between the measured case and the CFD predicted cases, which indicates that the distribution of static pressure is well modelled by these three turbulence models.

The well modelling of heat transfer coefficient $(h)$ distribution is necessary in the current optimization approach. Comparing the $h$ distributions among the measured data and the CFD predicted data by different turbulence models in Figure 4, the SST model shows much better performance in modelling different zones of $h$ distribution made by the lift-off lines [9]: the wedge shaped region of low heat transfer that extends into the mouth of the passage, the high rates of heat transfer downstream of the three dimensional separation line and the high heat transfer region between the lift-off line of the horseshoe vortex and the airfoil leading edge. Much better performance in modelling the values of $h$ near the airfoil trailing edge can be found in the case with SST model than the other two models. Though with CFD uncertainties, CFD results with the SST model are considered acceptable in the current study.

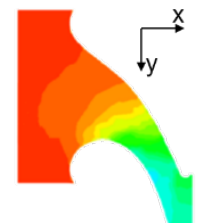

(a) Experiment

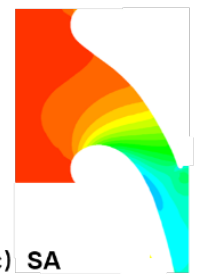

(c) SA
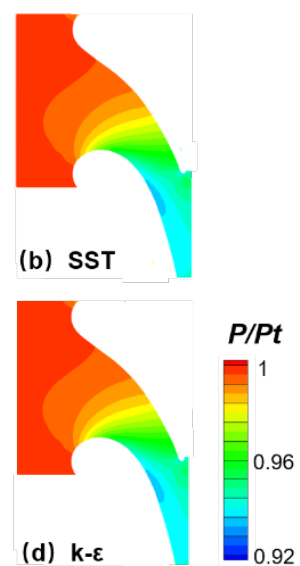

Fig. 3 Static pressure coefficient distribution on the endwall

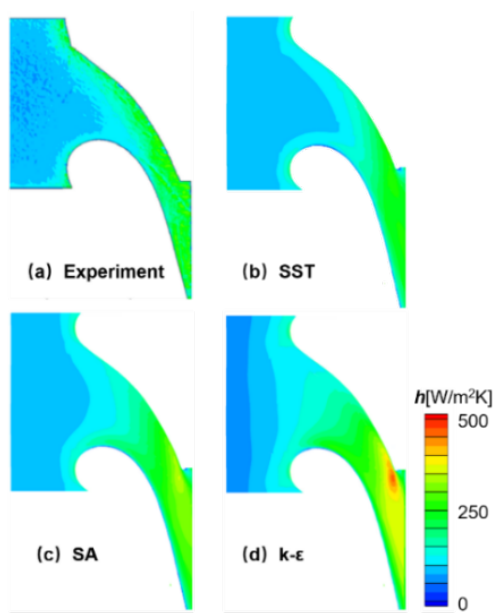

Fig. 4 heat transfer coefficient distribution on the endwall

\section{OPTIMIZATION METHODOLOGY}

\section{Optimization Process}

To find both improved aerodynamic performance of the passage and less total heat flux through the endwall, an optimization approach was employed. Figure 5 shows the flow chart of the optimization process. It contains a Genetic Algorithm (GA) [10] part, a CFD Database part and the Artificial Neural Network (ANN) [11] learning process part.

The strategy to determine the shape of the endwall from the parameters of the free points on the endwall is mentioned before (see figures 1). The CFD method used in the current optimization process is validated by experimental data (see Figures 3 and 4).

The optimization approach is a two-layer optimization process: the outer layer (the processes with blue arrows in Figure 5) optimization based on CFD evaluation in which the ANN models are trained using the CFD database and the optimal designs found in the inner layer are checked using CFD methods, and the inner layer (the processes with red arrows in Figure 5) optimization by GA optimization based on ANN model evaluation by which the optimal designs are quickly found.

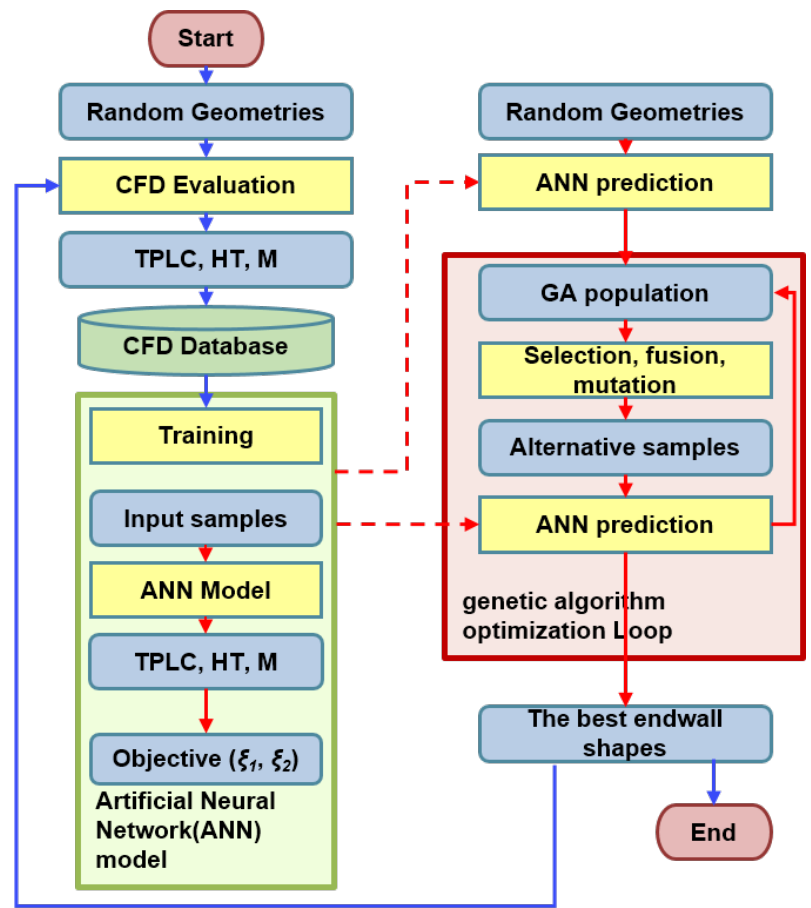

Fig. 5 Flow chart of the optimization process

A few steps are done in the current contoured endwall optimization process. Firstly, an initial CFD database are built by 780 samples generated using CFD simulations, each with a new randomly-chosen set of the 30 free points. These data sets are generated using a pseudo-random technique called "random among discrete levels." Which is suitable for a high number of variables [12]. With the values of the 30 free points as the input and the passage total pressure loss coefficient (TPLC), endwall total heat flux (HT, defined in Equation 3 below) and passage mass flowrate (M) values as the output, the initial ANN model is trained using the data in the initial CFD database. Secondly, the inner layer optimization is conducted by a GA approach based on the ANN model. GA mimics natural behaviors in terms of biological evolution in order to reach the best possible solution to a given problem. For the current multi-objective optimization, a nondominated sorting genetic algorithm II (NSGA-II) [13] is applied in the GA optimization process. NSGA-II uses an elitism based nondominated sorting method for ranking and sorting each individuals and uses a crowding distance approach in its section operator for 
keeping the diversity among the obtained Pareto optimal solutions. To start the GA optimization loop, an initial population with different sets of the free parameter values is generated and each individual of the population is solved using ANN model to evaluate the values of the objection functions ("Random geometries" in the red box in Figure 5, 50 individuals). The optimizing process for each reproduction cycle in the GA optimization step is done based on these geometry values, the objective function values and the ANN model. After 100 reproduction cycles, 4 optimized individuals are chosen as the optimized cases for the inner layer optimization in the current loop. Thirdly, the 4 optimized individuals are checked using CFD method to find much more accurate objective function values for them. Then, these objective function values of the newly achieved 4 optimization cases are compared with those of the cases from the previous optimization loops. If the optimized cases in the current loop achieve advantages in the objective function values to those from the previous loops, the optimal designs of the whole optimization process will be renewed by them and the Pareto front of the optimization will also be renewed. The CFD database is also enriched by these CFD cases and the ANN model used in the next optimization loop is re-trained by the data from the renewed database. In the current optimization, 400 optimization loops are done to find the optimal designs that meet the purpose of the optimization.

\section{Optimization Objectives}

The objective of the optimization process is to find the passage with non-axisymmetric contoured endwall design featuring both reduced aerodynamic loss in the passage and decreased total heat flux through the endwall surface. To make it possible, two objective functions, $\xi_{1}$ and $\xi_{2}$ were defined to represent the aerodynamic performance and the heat transfer characteristics as shown in Equation 1:

$$
\min \left(\xi_{1}, \xi_{2}\right)
$$

in which $\xi_{1}$ is defined as Equation 2 to represent the aerodynamic performance which expresses the relative difference between current aerodynamic loss values and the ideal condition values:

$$
\xi_{1}=w_{T P L C}\left(\frac{\overline{\overline{T P L C}}-0}{\overline{\overline{T P L C}}}\right)_{r e f}^{k_{T P L C}}
$$

in which the TPLC is the total pressure loss coefficient as defined in Equation 1. $w T P L C$ is the weight factor, which allows the scaling up or down of $\xi_{1}$ to make it comparable to $\xi_{2} . k_{T P L C}$ is the exponent of the objective function which is 2 in the current optimization process. 0 is set as the ideal condition of $\overline{\overline{T P L C}}$ and $\overline{\overline{T P L C}}_{\text {ref }}$ is set to be 1 as the reference.

The heat transfer characteristics is determined using the areaweighted integral of the heat transfer coefficient $(H T)$ to represent the total heat flux through the endwall, as defined in Equation 3:

$$
H T=\iint_{A} h d A
$$

where $h$ is the local heat transfer coefficient value on the endwall and $A$ is the area of the endwall. Based on Equation 4, the other objective function, $\xi_{2}$, which is defined to represent the total heat flux characteristics is defined as:

$$
\xi_{2}=w_{H T}\left(\frac{H T-0}{H T_{r e f}}\right)^{k_{H T}}
$$

in which the weight factor $w_{H T}$ is set to make the values of $\xi_{1}$ and $\xi_{2}$ comparable. The exponent factor $k_{H T}$ is set as 2 . The ideal condition of $H T$ value is 0 and $H T_{\text {ref }}$ value is set as the averaged $H T$ value among the cases in the database.

To maintain similar turbine reaction with the baseline case during optimization, the change of mass flow rate by endwall contouring must be kept within limits. Thus, a constraint of the mass flow rate value change between the current contoured-endwall passage with the un-contoured-endwall passage ranging from $0.5 \%$ to $0.5 \%$ is introduced into the optimization objectives. Any sample with mass flow rate out of the range of this mass flow rate constraint would not be chosen as the optimized case in the GA optimization process.

\section{OPTIMIZATION RESULTS AND OPTIMAL DESIGNS}

\section{Pareto Front and Optimal Designs}

The non-axisymmetric endwall shape optimization is carried out based on the current multi-objective optimization approach with two objective functions, $\xi_{1}$ and $\xi_{2}$, which are the objective function for aerodynamic performance and the objective function for heat transfer characteristics as described above. With optimization, Figure 6 shows the initial database generated by CFD evaluation, the optimized cases from each optimization loop and the final Pareto Front. As the optimization iterations, both of the two objective function values decrease (see the color change of the circles). Over 400 optimization loops were done to reach the current Pareto Front. Some representative individuals were chosen to be studied in detail, which are the baseline case and the three cases chosen from the Pareto Front (green asterisk for baseline case, cyan asterisk for case1, yellow asterisk for case 2 and magenta asterisk for case 3 in Figure 6). Among case1, case 2 and case 3 , case 1 is the case with the best aerodynamic performance but with the largest total heat flux value through the endwall, case 3 is the case with the worst aerodynamic performance but with the least total heat flux value through the endwall and case 2 is the case in between both for aerodynamic performance and total heat flux value.

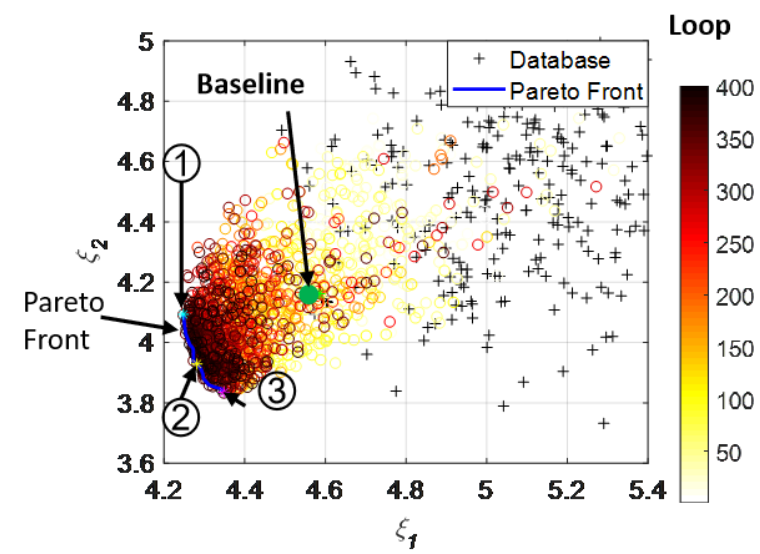

Fig. 6 Initial Database, Pareto Front, optimal cases in each loop (plotted as circles) and some individuals (green asterisk for baseline case, cyan asterisk for case1, yellow asterisk for case2 and magenta asterisk for case 3 )

The augment of TPLC, HT, $\overline{\bar{h}}$ and the total endwall area value for case 1 , case 2 and case 3 from the baseline case are listed in Table 2. These area-integrated and area-averaged values are calculated from $x / C_{a x}=-0.5$ to $x / C_{a x}=2$. It is interesting to find that though case 3 got the largest HT reduction value, it doesn't come with the smallest $\overline{\bar{h}}$ value among the three cases but the smallest endwall area augment value. Thus, it is important to note that when doing endwall optimization, endwall total area augment should be taken into account. 
Table 2 Aerodynamic and Heat Transfer Augment from Baseline Case

\begin{tabular}{c|ccc}
\hline Augment $/ \%$ & Case1 & Case2 & Case3 \\
\hline TPLC & -3.31 & -2.95 & -2.46 \\
\hline HT & -1.05 & -3.67 & -4.83 \\
\hline $\bar{h}$ & -2.51 & -5.58 & -5.02 \\
\hline Area & 1.49 & 2.02 & 0.20 \\
\hline
\end{tabular}

The endwall shapes of case1, case 2 and case 3 are shown in Figure 7 in which $\triangle \mathrm{R}$ is the local change of radial height from the baseline case (un-contoured endwall case) and Span is the spanwise size of the cascade. Though with different advantages in aerodynamic or heat transfer characteristics, the three cases are shaped in similar ways: big sunken zones from $x / C_{a x}=0.1$ to $x / C_{a x}=0.7$ or somehow further downstream which almost occupy the passage pitchwise from the vane pressure side to the vane suction side and small raised zones near the suction side in the aft part of the passage. The small raised area near the suction side could be a result for the constraint of the mass flowrate change to keep the throat area of the passage unchanged with the sunken zones in the middle of the passage. For case 1 and case2, there are very small raised zones around the vane pressure side near $x / C_{a x}=0.5$.

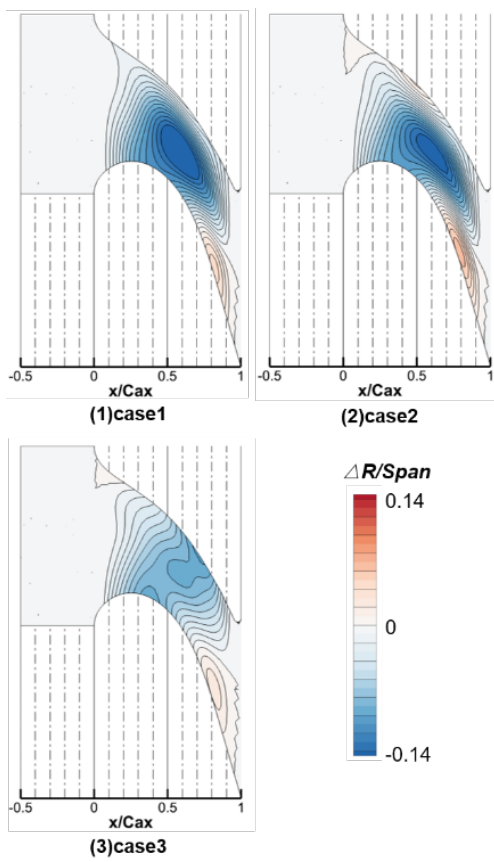

Fig. 7 Relative radial height distribution of the contoured endwalls: (1) case1 (2) case2 (3) case3

\section{Influence of Endwall Contouring on Aerodynamics}

Endwall contouring changes the pressure distribution, mainly the pressure gradients across the passage, to improve the aerodynamic performance inside of the passage. Figure 8 plots the static pressure coefficient distributions along several pitch-wise lines: $x / C_{a x}=0.1, x / C_{a x}=0.5$ and $x / C_{a x}=0.9$. In the fore part of the passage, the contoured endwalls acquired lower pressure near the airfoil suction side leading to larger pressure gradient across the passage. However, when it come downstream, for example, along the pitch-wise line of $x / C_{a x}=0.5$, the pressure values near the airfoil suction side are much larger than the baseline, which lower the pressure gradients across the passage. It is more complicated in the aft part of the passage for the contoured endwalls with a lower pressure zone across the passage perpendicular to the inviscid streamlines and a higher pressure zone downstream of the hill near the suction side: the pitchwise pressure gradient results into a "V" shape in which the bottom with smallest static pressure values are in the middle across the passage which enhanced the pressure gradients from the airfoil pressure side to the middle of the passage and from the airfoil suction side to the middle of the passage. This phenomenon is unlike what is conventionally expected, which is that the value of the static pressure near the airfoil pressure side should be lower than that of the baseline while the static pressure value near the airfoil suction side should be higher than that of the baseline.

Endwall contouring can also adapt the stream-wise pressure gradients, thus, change the flow acceleration performance. Figure 9 shows the static pressure coefficient distribution along the inviscid streamline paths for case 1 , case 2 , case 3 and the baselines. To obtain the inviscid streamline paths, an inviscid FLUENT [14] prediction of the inviscid streamlines on the endwall was done. Streamlines were released $1.5 C_{a x}$ upstream of the cascade from three pitchwise locations, so that the streamlines would pass through $\theta / \theta_{\text {pitch }}=0.25$, 0.50 and 0.75 at the inlet plane of the cascade. These streamlines are denoted as $0.25 \mathrm{P}, 0.50 \mathrm{P}$ and $0.75 \mathrm{P}$ in the current study. Generally, the contoured endwall cases achieved flatter pressure distributions along the inviscid streamline paths in the fore part of the passage upstream of $x / C_{a x}=0.5$ but much larger stream-wise pressure gradients in the accelerating region downstream of $x / C_{a x}=0.5$. In the fore part of the passage, there is even adverse pressure gradients along the $0.75 \mathrm{P}$ streamline path right downstream of the airfoil leading edge. The mild acceleration in the fore part of the passage results from the expansion of the passage by endwall contouring which can result in larger turning of streamlines around the endwall. The sharp acceleration in the aft part of the acceleration region resulted from the decreasing of the passage area there and also the curvature effect of the curved surface there. In the aft part of the passage, the lower pressure regions comparing to the baseline case perpendicular to the inviscid streamlines intensified the acceleration. In the recovery zone downstream of the low pressure locations due to the expansion of the passage, the contoured endwall cases acquired steeper adverse pressure gradient there, which can lead to larger curvature of streamlines there.

Comparing case 1 , case 2 and case 3 , the changes in pressure distribution from the baseline case among these three contoured endwall cases are similar with small differences. Normally, case 1 with best aerodynamic performance features the largest variations and case 3 features the smallest variations in most locations throughout the endwall.

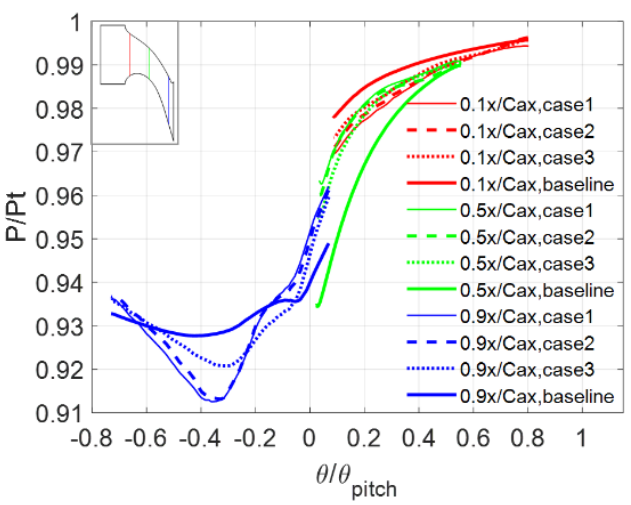

Fig. 8 static pressure coefficient distributions along pitchwise lines 


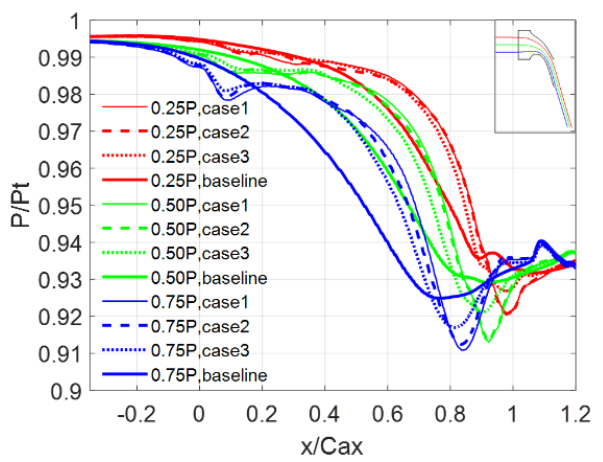

Fig. 9 static pressure coefficient distributions along inviscid streamlines

The limit streamlines on the endwalls for case1, case2, case 3 and the baseline case are plotted in Figure 10. The contoured endwalls in the current study still featured the classic endwall zones but with zone sizes and local streamline curvatures different from those of the baseline case. The suction side leg horseshoe vortexes reach the suction side surface of the airfoil at a more upstream position due to higher pressure gradient from the pressure side to the suction side and also due to the lack of acceleration by smaller stream-wise pressure gradient distributions. The pressure side leg horseshoe vortexes experience higher pitchwise pressure gradient when it goes through the fore part of the passage $\left(x / C_{a x}<0.15\right)$ but lower pitch-wise pressure gradient when it goes closer to the vane suction side $\left(x / C_{a x}>0.15\right)$. Meanwhile, they experience much smaller stream-wise pressure gradient especially downstream of $x / C_{a x}=0.2$ than that of the baseline case, thus weaker accelerations appear there. According to Figure 10, the locations of the pressure legs keep unchanged in the contoured endwall cases from the baseline case upstream of $x / C_{a x}=0.2$ but redirected to the suction side with larger streamline curvature downstream of $x / C_{a x}=0.2$. It indicates that the stream-wise pressure gradient matters more than the pitchwise pressure gradient when it comes to redirection of the pressure side leg horseshoe vortexes.
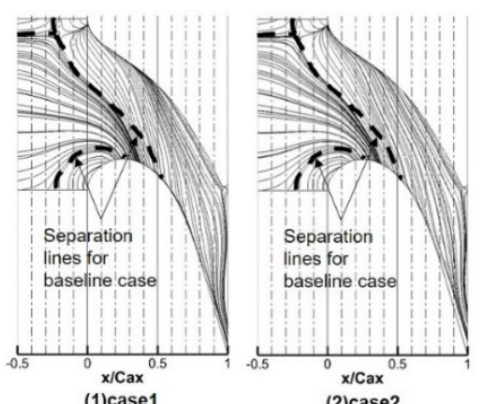

case2
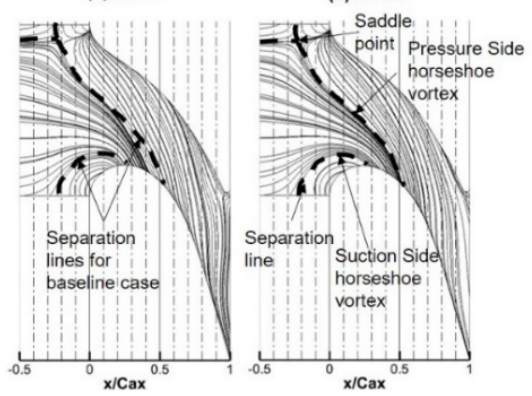

Fig. 10 Limit streamlines distributions on the endwall (1) case1.

(2) case2 (3) case3 (4) baseline

In the regions downstream of the lift-off line of the pressure side leg vortex, things come out in a different way. In the region near the pressure side upstream of $x / C_{a x}=0.5$, the streamlines featured larger angle to the axial direction (x direction) due to larger pitchwise pressure gradient values and lower stream-wise acceleration effects. When it comes to the region with higher stream-wise acceleration which is approximately downstream of $x / C_{a x}=0.5$, the streamlines are more straight in the contoured endwall cases than the baseline, especially near the airfoil suction side where the flow features relatively higher speed than that near the airfoil pressure side there. What's more, visible difference of streamline distributions can be found in the aft region of the passage. Due to larger adverse pressure gradient for the contoured endwall cases there, the flow decelerates more than that of the baseline case, and, with the larger pitchwise pressure gradient from the airfoil pressure side to the middle of the passage, the streamlines are with larger angles to the axial direction. With larger positive pressure gradients from the suction side to the middle of the passage near the trailing edge of the airfoil suction side for the contoured endwall cases, the streamlines tend to move away from the airfoil suction side, forming a region with streamlines parallel to the suction side profile there.

As the changes of cases 1,2 and 3 in static pressure distribution and streamline distributions from the baseline case are similar, case 1 is chosen as an example to explore the difference of flow fields inside of the passages from the baseline case. Figure 11 shows the distributions of stream-wise vorticity and static pressure coefficients on the plane of $x / C_{a x}=0.75$ for case 1 and baseline. The size of the passage vortex is much larger for case 1 than that of the baseline due to the lack of acceleration near the pressure side in the fore and mid part of the passage for case1. Wall vortexes arises near the airfoil suction side downstream of the location where the pressure side horseshoe vortexes reach the suction side and turn into the passage vortex. In addition, the pressure gradient from the midpitch of the passage to the airfoil suction side enlarges the passage vortex shown as the black arrow in Figure 11(3).

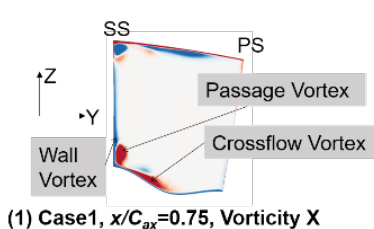

SS

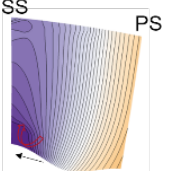

(3) Case1, $x / C_{a x}=0.75, P / P_{t}$

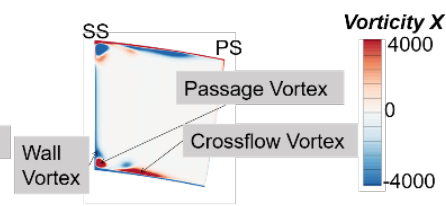

(2) baseline, $x / C_{a x}=0.75$, Vorticity $x$

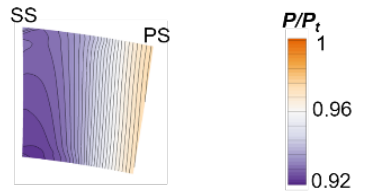

(4) baseline, $x / C_{a x}=0.75, P / P$

Fig. 11 Stream-wise vorticity distribution and static pressure

coefficient distribution: (1) case $1, x / C_{a x}=0.75$, Vorticity X. (2) baseline, $x / C_{a x}=0.75$, Vorticity X. (3) case $1, x / C_{a x}=0.75$, P/Pt. (4) baseline, $x / C_{a x}=0.75, \mathrm{P} / \mathrm{Pt}$.

Stream-wise vorticity and static pressure coefficient distributions on the plane of $x / C_{a x}=0.96$ are plotted in Figure 12 to look into the flow field in the aft part of the passage. The wall vortex and passage vortex for case 1 are with a larger distance from the hub than that of the baseline as seen on the plane of $x / C_{a x}=0.5$ in Figure 12 due to the steep adverse stream-wise pressure gradient and also the small "hill" geometry near the airfoil suction side right upstream of the $x / C_{a x}=0.96$ plane. There are counter rotating corner vortexes found in the suction side corner region of case1: with positive pressure gradient around the airfoil suction side next to the hub, a vortex with positive vorticity $\mathrm{X}$ is generated there under the general corner vortex induced the passage vortex and the negative pressure gradient around the airfoil suction side in the higher span-wise location. Also, with positive pressure gradient from the airfoil suction side to the middle of the passage (see Figure 8, pressure distribution across the passage, $x / C_{a x}=0.9$ ), negative vorticity $\mathrm{X}$ are generated inside of the boundary layer around the hub in the corner region. 


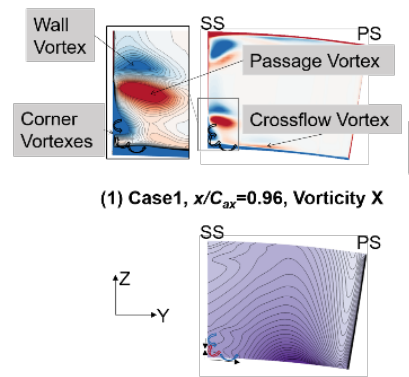

(3) Case1, $x / C_{a x}=0.96, P / P_{t}$

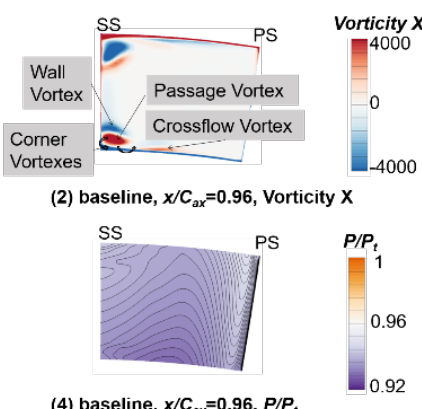

(4) baseline, $x / C_{a x}=0.96, P / P$
Fig. 12 Streamwise vorticity distributions and static pressure coefficient distributions: (1) case $1, x / C_{a x}=0.96$, Vorticity X. (2) baseline, $x / C_{a x}=0.96$, Vorticity X. (3) case $1, x / C_{a x}=0.96$, P/Pt. (4) baseline, $x / C_{a x}=0.96, \mathrm{P} / \mathrm{Pt}$.

Though the vortexes structures near the shroud are not changed much, the size and intensity of the vortexes there are still adapted. The difference of the flow field in the passage results in visible difference for vorticity $\mathrm{X}$ distributions in the downstream locations of the cascade between case 1 and the baseline case, e.g., the streamwise vorticity distributions on the plane of $x / C_{a x}=1.06$ plotted in Figure 13. The differences of the flow field not only exist in the lower half part of the passage, which is the difference on wall vortex, passage vortex and corner vortexes, but also exists near the shroud, which can be found in the size of the passage vortex near the shroud.

Figure 14 shows the distribution of TPLC for casel and the baseline case on the plane of $x / C_{a x}=1.25$. With larger passage vortexes near the shroud, the loss core near the shroud for the baseline is larger than that of case1. More obvious difference of the TPLC distributions can be found in the lower part of the passage: there are two loss cores for Case1, one at higher span-wise location resulted from the passage vortex and the wall vortex, the other one at lower span-wise location arisen from the counter rotating corner vortexes. However, for the baseline case, only one large and strong loss core exists for the counter rotating passage vortex and the wall vortex. Pitch-wise mass flow weight averaged TPLC distributions for the plane of $x / C_{a x}=1.25$ are plotted for cases1, 2, 3 and the baseline case in Figure 15. In the lower half span of the passage, the two loss cores for case 1 are at higher span-wise locations than those of case 2 and case 3 , which is due to larger adaption of the endwall shape for case 1 than that of the other two cases. Smaller TPLC values are found for case 1, 2, 3 than that of the baseline in the higher half span due to the changes in passage vortexes there.

Table 3 lists the mass flow weight averaged TPLC on the plane of $x / C_{a x}=1.25$, and on the upper/lower half span of this plane for case 1 , case 2 , case 3 and the baseline case. Though case 1 achieved smallest $\overline{\overline{T P L C}}$ value at the outlet of the CFD domain, it doesn't perform better than the other two contoured endwall cases on the plane of $x / C_{a x}=1.25$. The mixing of the vortexes downstream of the plane of $x / C_{a x}=1.25$ are changing the aerodynamic performance for each sample. Comparing the lower half and higher half of the passage, the changes in the flow field both in the upper half span and the lower half span are reducing the aerodynamic loss, in which the improvement in the upper span is more significant and cannot be ignored.

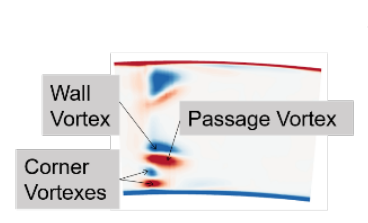

(1) Case1, $x / C_{a x}=1.06$, Vorticity $x$

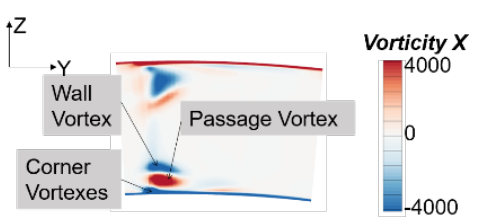

(2) baseline, $x / C_{a x}=1.06$, Vorticity $X$
Fig. 13 Streamwise vorticity distribution: (1) case1, $x / C_{a x}=1.06$, Vorticity X. (2) baseline, $x / C_{a x}=1.06$, Vorticity X

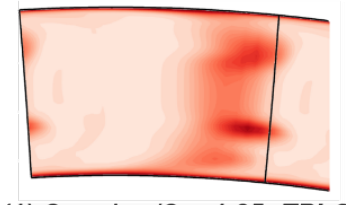

(1) Case1, $x / C_{a x}=1.25, T P L C$

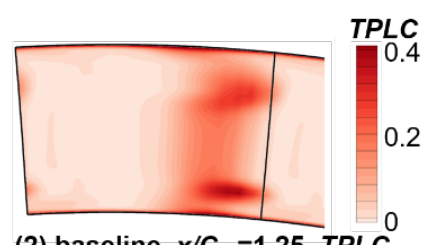

(2) baseline, $x / C_{a x}=1.25, T P L C$
Fig. 14 Total pressure loss coefficient distributions: 1) case1, $x / C_{a x}=1.25$ (2) baseline, $x / C_{a x}=1.25$

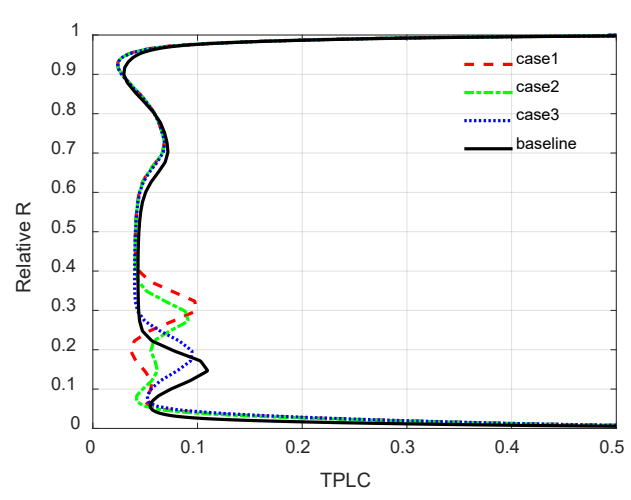

Fig. 15 Pitchwise massflow weight averaged TPLC distributions for the plane of $x / C_{a x}=1.25$

Table 3 Mass flow weight averaged TPLC

\begin{tabular}{c|cccc}
\hline & Case1 & Case2 & Case3 & Baseline \\
\hline$\overline{\overline{T P L C}}$ & 0.0602 & 0.0597 & 0.0599 & 0.0634 \\
\hline$\overline{\overline{T P L C}}_{\text {span0-50\% }}$ & 0.0661 & 0.0656 & 0.0659 & 0.0686 \\
\hline$\overline{\overline{T P L C}}_{\text {span50-100\% }}$ & 0.0542 & 0.0539 & 0.0540 & 0.0578 \\
\hline
\end{tabular}

\section{Influence of Endwall Contouring on Heat Transfer}

The change of the flow field around the hub due to endwall contouring can change the heat flux distributions through the hub. Figure 16 plots the distributions of $h$ augment on the hub for case 1 , case 2 and case 3 . A positive $h$ augment zone is in the fore part of the passage near the suction side for the current three contoured endwall cases. With deep sunken area in the middle of the passage for case 1 and case2, the $h$ augment zones are separated as the zone near the airfoil pressure side and the zone near the airfoil suction side. Near the airfoil pressure side, there is zones with positive $h$ augment in the fore and mid part of the passage but negative $h$ augment near the airfoil trailing edge for case 1 and case2. It is different for case 3 : negative $h$ augment appears near the pressure side upstream of $x / C_{a x}=0.75$ but a zone with heat transfer elevation right downstream of $x / C_{a x}=0.75$. The positive $h$ augment zone near the pressure side in the fore part of the passage for case 1 and case 2 is related to the small raised zones around the vane pressure side near $x / C_{a x}=0.5$. For the other half part of the endwall that near the airfoil suction side, there is a reduced $h$ zone with large size in the fore part of the passage and an enhanced $h$ zone with small size in the middle aft part of the passage. Near the trailing edge, $h$ is reduced for the contoured endwall cases. Due to the larger distance of the passage vortex from the hub and the smaller mixing made by the corner vortexes in the contoured endwall passages comparing to the flow field of the baseline passage in the regions downstream of the airfoil trailing edge, the contoured endwalls feature $h$ reducing there. 


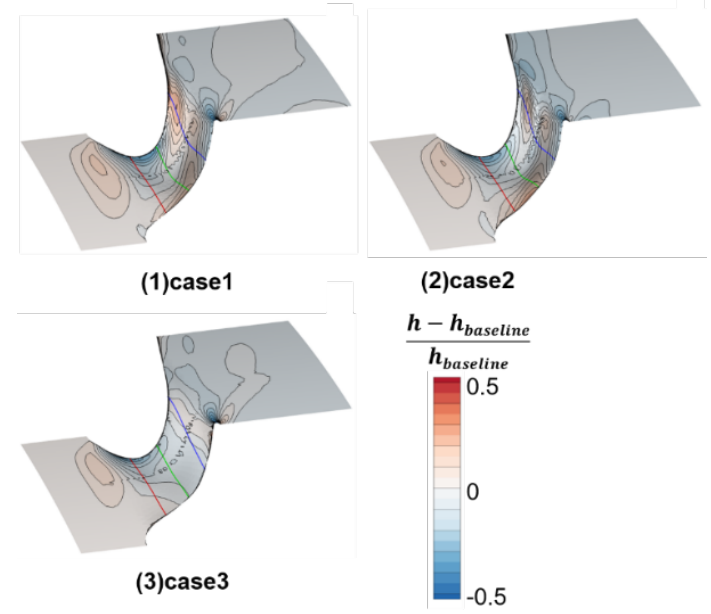

Fig. 16 Heat transfer coefficient augment distribution on the hub of case 1 , case 2 and case 3

Figure 17 plots the distribution of $h$ on the hub for of case1, case 2 , case 3 and the baseline along inviscid streamline paths $0.25 \mathrm{P}$ and $0.75 \mathrm{P}$. The $h$ along $0.25 \mathrm{P}$, which is near the airfoil pressure side, is slightly elevated with $x / C_{a x}$ smaller than 0.8 but remarkably reduced downstream of $x / C_{a x}=0.8$ until the location $0.5 C_{a x}$ downstream of the airfoil trailing edge. Near the airfoil suction side, the $h$ is reduced in the fore part of the passage, which is $x / C_{a x}$ smaller than 0.6 but featured larger $h$ in the range from $x / C_{a x}=0.6$ to $x / C_{a x}=0.85$. Smaller $h$ is acquired downstream of $x / C_{a x}=0.85$ until far downstream of the airfoil trailing edge. Though enhanced $h$ near the suction side in the aft part of the passage, the high $h$ near the airfoil pressure side around the leading edge is reduced by endwall contouring.

The contoured-endwall passages in the current study still featured the classic endwall zones but with zone sizes and local streamline curvatures different from those of the baseline case, which makes the adaption of local velocity distribution becomes a solution to change the heat transfer coefficient distributions. Figure 18 plots the non-dimensional velocity distributions on the planes of $x / C_{a x}=0.25,0.5$ and 0.75 for case 1 , case 2 , case 3 and the baseline case. It is clear to see that the lower velocity near the suction side at the fore part of the passage for the contoured endwall cases accounts for the reduced heat transfer coefficient there. And the same reason of the velocity change in the passage can be used to explain the heat transfer enhanced zone and heat transfer reduced zone in the downstream part of the passage. The vortex structures inside of the passage are adapted but not that velocity adaption becomes a solution to change the $h$ values.

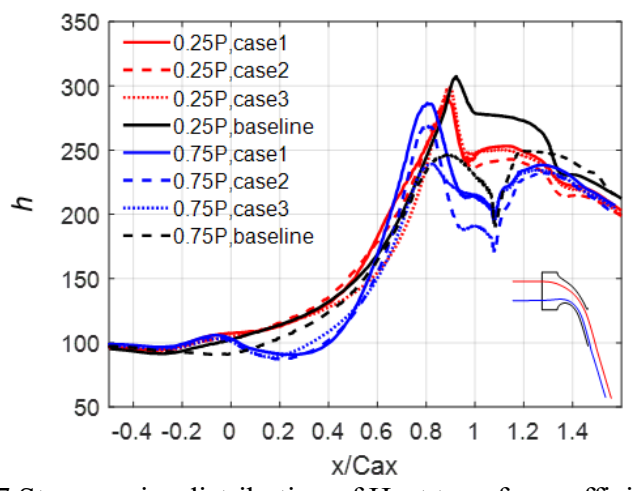

Fig. 17 Stream-wise distribution of Heat transfer coefficient on the hub for of case 1 , case 2 and case 3 along inviscid streamline paths

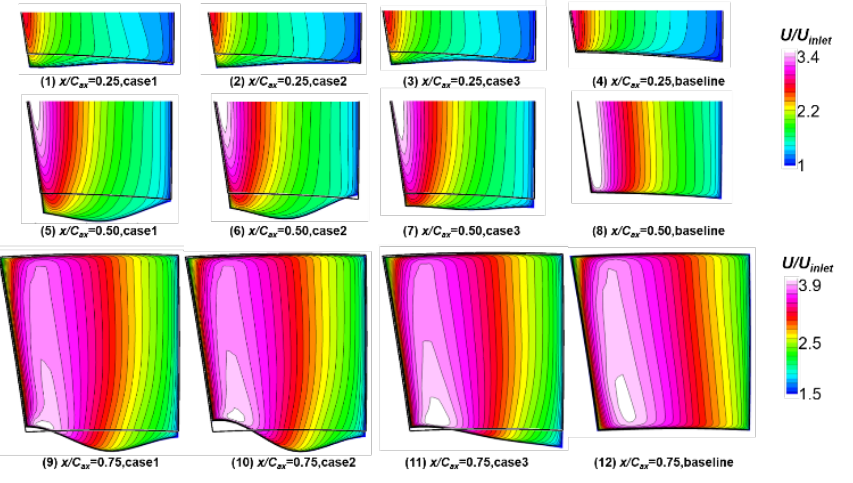

Fig. 18 non-dimensional velocity distributions on several Planes $(\mathrm{x} / \mathrm{Cax}=0.25,0.50$ and 0.75$)$

\section{CONCLUSION}

To find non-axisymmetric endwall designs with both improved aerodynamic performance and heat flux benefits, a multi-objective optimization approach is designed and carried out. Several optimized cases are found as the Pareto Front of the current optimization process. The sample with advantages in aerodynamic performance features $3.31 \%$ decrease in total pressure loss coefficient value and the sample with advantages in total heat flux values through the endwall obtained $4.83 \%$ total heat flux reduction.

Three typical cases with scattered aero and heat transfer performances are chosen for further study. It was found that the three optimized cases feature similar contouring method, which is big sunken area in the fore part of the passage and a small raised area in the aft part of the area near the airfoil suction side. The difference on aerodynamic performance and heat flux features among the optimized cases are only due to slight difference in endwall shaping.

The change in the vortexes near the hub is mainly due to the adaption in the stream-wise acceleration features. The improvement in aerodynamic performance is the combination of the adapted vortexes near the contoured hub and also the change of the features of the vortexes near the un-contoured shroud.

The modification of the total heat flux is determined by the change in heat transfer coefficient and the change in total area of the endwall. The case gives consideration to both heat transfer coefficient augment and total endwall area can be the case with improved total heat flux features.

\section{ACKNOWLEDGEMENT}

The authors would like to acknowledge the financial support by National Science and Technology Major Project of China (2017-III0009-0035) and National Natural Science Foundation of China (No. 51706116).

\section{REFERENCES}

[1] Giampaolo, T. (2002). Gas turbine handbook: principles and practice. Fairmont Press.

[2] Harvey, N. W., Rose, M. G., Taylor, M. D., Shahpar, S., Hartland, J., \& Gregory-Smith, D. G. (2000). Nonaxisymmetric turbine end wall design: part I-threedimensional linear design system. Journal of Turbomachinery, 122(2), 278-285.

[3] Hartland, J. C., Gregory-Smith, D. G., Harvey, N. W., \& Rose, M. G. (2000). Nonaxisymmetric turbine end wall design: part II-experimental validation. Journal of turbomachinery, 122(2), 286-293.

[4] Praisner, T. J., Allen-Bradley, E., Grover, E. A., Knezevici, D. C., and Sjolander, S. A., 2007, "Application of NonAxisymmetric Endwall Contouring to Conventional and High-Lift Turbine Airfoils," Proc. ASME Turbo Expo 2007, Montreal, Canada, May 14-17, ASME Paper No. GT2007- 
27579.

[5] Lynch, S. P., Sundaram, N., Thole, K. A., Kohli, A., \& Lehane, C. (2011). Heat transfer for a turbine blade with nonaxisymmetric endwall contouring. Journal of Turbomachinery, 133(1), 011019.

[6] Winkler, S. (2016). Endwall contouring using numerical optimization in combination with the ice formation method.

[7] F.C. Park, B. Ravani, Bezier Curves on Riemannian Manifolds and Lie Groups with Kinematics Applications, Trans. ASME, J. Mech. Des. 117 (1995) 37-40. doi:10.1115/1.2826114.

[8] Li, X., Ren, J., \& Jiang, H. (2017). Experimental Investigation of Endwall Heat Transfer with Film and Impingement Cooling. Journal of Engineering for Gas Turbines and Power, 139(10), 101901.

[9] Friedrichs, S. (1997). Endwall film-cooling in axial flow turbines (Doctoral dissertation, University of Cambridge).

[10] Mitchell M. An introduction to genetic algorithms [M]. MIT press, 1998.

[11] Yegnanarayana B. Artificial neural networks [M]. PHI Learning Pvt. Ltd., 2009.

[12] Van Der Merwe B B. Design of a centrifugal compressor impeller for micro gas turbine application [D]. Stellenbosch: Stellenbosch University, 2012.

[13] Deb K, Agrawal S, Pratap A, et al. A fast elitist non-dominated sorting genetic algorithm for multi-objective optimization: NSGA-II[C]//International conference on parallel problem solving from nature. Springer, Berlin, Heidelberg, 2000: 849858.

[14] Fluent Inc., FLUENT (version 6.3.26), Lebanon, NH. 